(1)

CrossMark

\title{
Mucolytics for COPD: negotiating a slippery slope towards proof of efficacy
}

\author{
Shawn D. Aaron \\ Affiliation: The Ottawa Hospital Research Institute, University of Ottawa, Ottawa, Canada.
}

Correspondence: Dr S.D. Aaron, The Ottawa Hospital, General Campus, 501 Smyth Road, Ottawa, Ontario, K1H 8L6, Canada. E-mail: saarondohri.ca

@ERSpublications

Clinical trials have thus far not conclusively demonstrated that mucolytics are effective therapies for COPD http://ow.ly/wbLe30eKB7H

Cite this article as: Aaron SD. Mucolytics for COPD: negotiating a slippery slope towards proof of efficacy. Eur Respir J 2017; 50: 1701465 [https://doi.org/10.1183/13993003.01465-2017].

Mucolytics are medicines that modulate mucus production and decrease mucus viscosity. Their mucus thinning effects may improve mucociliary transport and thereby facilitate expectoration of mucus out of the distal bronchial tree [1]. Improved clearance and expectoration of mucus can prevent mucus plugging of small airways and may theoretically improve gas exchange and lessen the probability of post-obstructive infection. Through these complementary effects, mucolytics could potentially play a role in reducing exacerbations in chronic obstructive pulmonary disease (COPD) [2].

Mucolytic therapies have shown clear benefits in clinical trials involving patients with cystic fibrosis (CF) $[3,4]$; however, in contrast, they have shown harmful or mixed effects in clinical trials involving patients with COPD. The most well known mucolytics are inhaled dornase alfa (recombinant DNAse), oral $\mathrm{N}$-acetylcysteine and inhaled hypertonic saline. A Phase-III trial of inhaled dornase alfa in patients hospitalised with COPD was stopped early because of a trend towards higher 90-day mortality rates in patients treated with the active drug [5]. A similar clinical trial of dornase alfa in patients with non-CF bronchiectasis (many of whom also had COPD) showed potential harm, as more frequent pulmonary exacerbations and greater decline in forced expiratory volume in $1 \mathrm{~s}(\mathrm{FEV} 1)$ decline were observed in patients who received inhaled dornase alfa compared to those receiving a placebo [6].

The data supporting $\mathrm{N}$-acetylcysteine for prevention of acute exacerbations of COPD is similarly mixed. A clinical trial published by Zheng et al. [7] studied 1006 Chinese patients with moderate to severe COPD who were randomised either for treatment with oral $\mathrm{N}$-acetylcysteine (600 mg twice daily) or with placebo for 1 year. After 1 year, $\mathrm{N}$-acetylcysteine was found to decrease the relative risk of acute exacerbations of COPD by $22 \%$ relative to placebo. However, the Zheng study has been criticised because exacerbations were assessed symptomatically by diary cards and were mostly unreported and mild in severity [8]. A well-designed European clinical trial enrolled 523 patients with COPD and randomly assigned them to daily $\mathrm{N}$-acetylcysteine or placebo treatment. Over a 3-year follow-up period the number of exacerbations requiring medical attention did not differ between groups (hazard ratio 0.99; 95\% CI 0.89-1.10) and the authors concluded that $\mathrm{N}$-acetylcysteine was ineffective at preventing moderate or severe exacerbations of COPD [9].

Erdosteine is an oral mucolytic that contains two blocked sulfhydryl groups which are released following first-pass metabolism. The active metabolites of erdosteine exhibit mucolytic and free radical scavenging activity [10] and previous small clinical trials have suggested some benefit in treating acute exacerbations

Received: July 192017 | Accepted: July 202017

Conflict of interest: None declared.

Copyright @ERS 2017 
of COPD with erdosteine [11, 12]. In this issue of the European Respiratory Journal, DAL NEGRO et al. [13] report results from a Phase-III controlled clinical trial that enrolled patients with GOLD stage II-III COPD and randomised them to receive erdosteine (300 $\mathrm{mg}$ twice daily) or a placebo for 12 months in addition to their usual COPD therapy. The primary outcome was the number of acute exacerbations (mild, moderate or severe) during the study. The study met its primary outcome in that erdosteine reduced the annual rate of COPD exacerbations by $19 \%$ compared to the placebo. Additional analysis also suggested that erdosteine decreased the duration of exacerbations.

Is this most recent erdosteine clinical trial a "slam dunk" and should we be prescribing erdosteine to our COPD patients who experience frequent exacerbations? Unfortunately, a deeper analysis of the study results suggests concerns. The most obvious concern is that although overall exacerbation rates were reduced with erdosteine, this effect was entirely driven by a reduction in mild exacerbations reported by patients on their home symptom diary cards. In fact, the annual rate of moderate or severe COPD exacerbations was $13 \%$ higher in the group treated with erdosteine compared to placebo and the increased incidence of moderate/severe exacerbations in those treated with erdosteine barely escaped statistical significance $(\mathrm{p}=0.054)$. Moderate or severe exacerbations are economically and clinically important since they entail physician visits, medication prescriptions and/or hospitalisations and they are associated with a greater risk of morbidity and mortality [14]. Given that erdosteine does not protect against these events, its benefits become much less compelling.

What about exacerbation duration? The investigators reported that in patients who experienced exacerbations the duration of exacerbation events was reduced by $24 \%$ by erdosteine treatment. Unfortunately, the definition of exacerbation duration used in this study was somewhat complicated and makes interpretation of the study results difficult. For mild events the end of the exacerbation event was recorded when the patients' symptoms returned to their previous stable value. Conversely, for moderate events, the investigators determined the end of an episode as the date when exacerbation treatment ended and for severe events the end of an episode was determined when the patient was discharged from hospital. One could easily imagine a study patient who had a severe exacerbation and was admitted to hospital for 1 day but who continued to have increased symptoms above baseline for 15 days. This patient would count as having an exacerbation duration of 1 day for study purposes. Alternatively, a patient with a mild exacerbation who had 5 days of increased symptoms and who self-treated at home with bronchodilators would have an exacerbation duration of 5 days. Unfortunately this means that in reality comparing the duration of all exacerbation events lumped together, as was done in this trial, is not valid.

In contrast to the evidence available in CF, the results of the DAL NEGRO study and previous studies of mucolytics do not provide compelling evidence to support prescription of mucolytics to patients with COPD. Perhaps the problem is that COPD-related studies of mucolytics have not restricted study entry to certain patient phenotypes, such as those with chronic cough and sputum, who might benefit more from mucolytic therapy. In any case, we can't at this point claim that the DAL NEGRO study has "coughed up a winning result". To date mucolytics have not yet climbed the slippery slope towards proof of efficacy in COPD and their routine prescription to patients with COPD is not yet warranted.

\section{References}

1 Balsamo R, Lanata L, Egan CG. Mucoactive drugs. Eur Respir Rev 2010; 19: 127-133.

2 Tarrant BJ, Le Maitre C, Romero L, et al. Mucoactive agents for chronic, non-cystic fibrosis lung disease: a systematic review and meta-analysis. Respirology 2017; 22: 1084-1092.

3 Elkins MR, Robinson M, Rose BR, et al. A controlled trial of long-term inhaled hypertonic saline in patients with cystic fibrosis. N Engl J Med 2006; 354: 229-240.

4 Fuchs HJ, Borowitz DS, Christiansen DH, et al. Effect of aerosolized recombinant human DNase on exacerbations of respiratory symptoms and on pulmonary function in patients with cystic fibrosis. The Pulmozyme Study Group. N Engl J Med 1994; 331: 637-642.

5 Genentech halts Pulmozyme COPD trial. London, The Pharma Letter, 1995. www.thepharmaletter.com/article/ genentech-halts-pulmozyme-copd-trial

6 O'Donnell AE, Barker AF, Ilowite JS, et al. Treatment of idiopathic bronchiectasis with aerosolized recombinant human DNase I. rhDNase Study Group. Chest 1998; 113: 1329-1334.

7 Zheng JP, Wen FQ, Bai CX, et al. Twice daily N-acetylcysteine $600 \mathrm{mg}$ for exacerbations of chronic obstructive pulmonary disease (PANTHEON): a randomised, double-blind placebo-controlled trial. Lancet Respir Med 2014; 2: $187-194$.

8 Turner RD, Bothamley GH. N-Acetylcysteine for COPD: the evidence remains inconclusive. Lancet Respir Med 2014; 2: e3.

9 Decramer M, Rutten-van Mölken M, Dekhuijzen PN, et al. Effects of N-acetylcysteine on outcomes in chronic obstructive pulmonary disease (Bronchitis Randomized on NAC Cost-Utility Study, BRONCUS): a randomised placebo-controlled trial. Lancet 2005; 365: 1552-1560.

10 Dal Negro RW, Visconti M, Micheletto C, et al. Changes in blood ROS, e-NO, and some pro-inflammatory mediators in bronchial secretions following erdosteine or placebo: a controlled study in current smokers with mild COPD. Pulm Pharmacol Ther 2008; 21: 304-308. 
11 Dal Negro RW, Visconti M, Turco P. Efficacy of erdosteine 900 versus 600-mg/day in reducing oxidative stress in patients with COPD exacerbations: results of a double blind, placebo-controlled trial. Pulm Pharmacol Ther 2015. 33: 47-51.

12 Moretti M, Fagnani S. Erdosteine reduces inflammation and time to first exacerbation postdischarge in hospitalized patients with AECOPD. Int J Chron Obstruct Pulmon Dis 2015; 10: 2319-2325.

13 Dal Negro RW, Wedzicha JA, Iversen $M$, et al. Effect of erdosteine on the rate and duration of COPD exacerbations: the RESTORE study. Eur Respir J 2017; 50: 1700711.

14 Hartl S, Lopez-Campos JL, Pozo-Rodriguez F, et al. Risk of death and readmission of hospital-admitted COPD exacerbations: European COPD audit. Eur Respir J 2015; 47: 113-121. 